\title{
Granular Dynamic Simulations of Depositing Materials: Two-Dimensional Approach
}

\begin{abstract}
Mohamad Hasan*
Department of Mathematics, Faculty of Mathematics and Natural Science, Universitas Jember, Indonesia. ${ }^{*}$ Corresponding author. Email: hasan.fmipa@unej.ac.id

ABSTRACT

During the deposition process, many factors play a role in the dynamics of the system including materials' characteristics and media onto which the materials dropped. The stick-slip model has been applied to simulate the depositions of polydisperse granular materials. As the size of the materials varied, and hence the criteria for colliding materials, the dynamics and the structures of the resulting systems could be different. The research aims are to investigate the dynamics of the deposition of polydisperse materials and the structures of the resulting piles. The results show that during the deposition process, internal landslide plays an important role, but surface avalanche is not the main mechanism as observed in monodisperse materials. In addition, the pile structures are not close-packed, and the force networks are not dominated by diamond shapes.
\end{abstract}

Keywords: Granular dynamics, Deposition, Surface avalanche, Landslide, Force networks.

\section{INTRODUCTION}

When we deposit materials into a surface or onto a container, early dropped materials may be stable (stick) after undergoing some dynamics due to the role of friction force. Those stable materials can be destabilized (slip) due to the collision (contact) with other materials. Hasan and van Opheusden [1] have developed collision force model that is able to mimic "stick-slip" phenomena. The particles collision and their forces are schematically shown in Figure 1.

Figure 1a illustrates the collision forces of particles $i$ and $\mathrm{j}$, and particle and the surface (medium). The forces consist of normal and tangential (friction) forces. In [1], the normal force is modeled by a Hookean spring and damper

$\mathbf{f}_{\mathrm{n}}=-\left(k_{\mathrm{n}} \boldsymbol{\delta}_{\mathrm{n}}+\gamma_{\mathrm{n}} \mathbf{v}_{\mathrm{n}}\right)$,

where $\boldsymbol{\delta}_{\mathrm{n}}$ and $\mathbf{v}_{\mathrm{n}}$ are the displacements (overlap between particles) and the relative velocity in the normal direction, and $k_{\mathrm{n}}$ and $\gamma_{\mathrm{n}}$ are the normal elastic and damping constants. For tangential force, the model applies two different coefficients of friction, static $\left(\mu_{\mathrm{s}}\right)$ and dynamic $\left(\mu_{\mathrm{d}}\right)$, and an " $\varepsilon$-criterion" as a threshold for the transition from dynamic to static motions. The tangential force is then modeled by

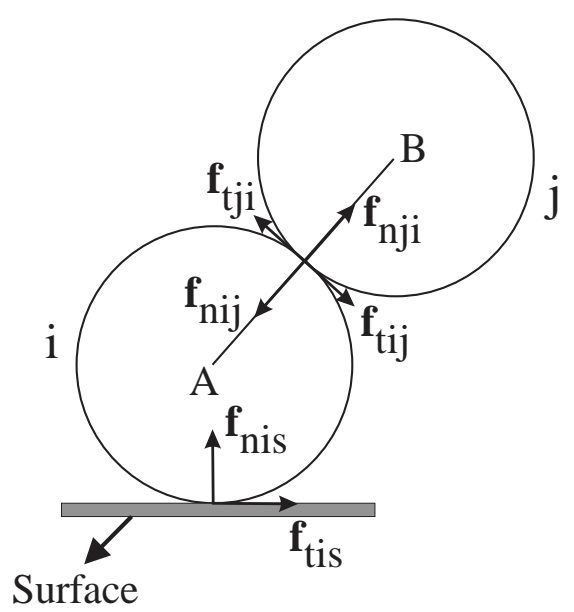

(a)

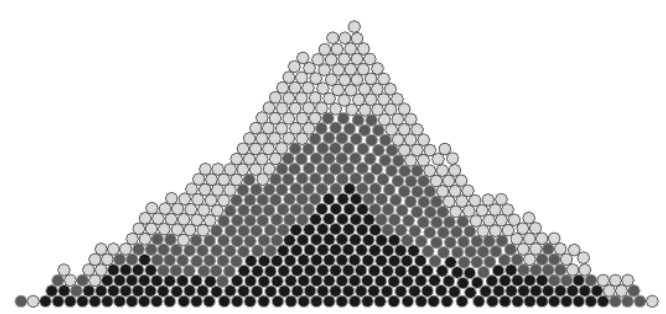

(b)

Figure 1 (a). Collision forces, and (b) Pile formation (discs with dark color dropped first). 
$\mathbf{f}_{\mathrm{t}}= \begin{cases}-\left(k_{\mathrm{t}} \boldsymbol{\delta}_{\mathrm{t}}+\gamma_{\mathrm{t}} \mathbf{v}_{\mathrm{t}}\right), & \left|\mathbf{v}_{\mathrm{t}}\right| \leq \varepsilon(\text { static mode }) \\ -\hat{\mathbf{v}}_{\mathrm{t}} \mu_{\mathrm{d}}\left|\mathbf{f}_{\mathrm{n}}\right|, & \left|\mathbf{v}_{\mathrm{t}}\right|>\varepsilon(\text { dynamic mode })\end{cases}$

where $\boldsymbol{\delta}_{\mathrm{t}}=\int_{t_{0}} \mathbf{v}_{\mathrm{t}}(\tau) d \tau$ is the total tangential displacement built up during the time duration $t-t_{0}, \mathbf{v}_{\mathrm{t}}$ is the relative velocity in the tangential direction, $k_{\mathrm{t}}$ and $\gamma_{\mathrm{t}}$ are the tangential elastic and damping constants, and the cap means an associated unit vector, indicating the direction only. The tangential force model (2) is similar to the model proposed by Brendel and Dippel [2], and Matuttis et al [3], but, in addition, we applied " $\varepsilon-$ criterion", in (2), $t_{0}$ is the time at which the quasi-static regime engages. The investigation by Hasan and van Opheusden [4] showed that the model does not suffer from oscillatory motion at the static mode by applying critical damping, i.e., $\gamma_{\mathrm{t}}=2 \sqrt{ }\left(m k_{\mathrm{t}}\right)$, where $m$ is particle mass.

Computer simulations of depositing monodisperse materials (materials size are the same) using this model had successfully produced piles as expected, and one of which is shown in Figure 1b. The dynamic (pile formation) of this system is dominated by surface avalanches and internal landslides. The details of pile formations and their structures are influenced by the characteristics of the materials such as elasticity and surface roughness.

Monodisperse systems are simple with respect to the deployment of numerical procedure, since throughout the system the radius of materials is constant, and hence the formula for calculating normal displacement is also constant, i.e.,

$\boldsymbol{\delta}_{\mathbf{n} i j}=\left(d_{i j}-\sigma\right) \hat{\mathbf{n}}$,

where $\sigma$ and $d_{i j}$ are the material diameter and the distance between materials $\mathrm{i}$ and $\mathrm{j}$ respectively. For polydisperse systems, equation (3) is no longer valid since the materials' diameters vary. Furthermore, the number of possible collisions for each material may be greater than 6 , which is not the case for monodisperse systems. Consequently, the numerical procedure for polydisperse systems are more complex than for monodisperse systems, and hence the previous code should be modified. As a result, the mechanism of pile formation and the structure of the resulting pile between the two systems could be different.

In this paper, the discussion is focused on the dynamic behavior of the deposition process of polydisperse materials, the structure of the resulting piles, and their force networks. The comparisons of the results of monodisperse and polydisperse systems are also described.

\section{METHODOLOGY}

The collision force models in equations (1) and (2) are also used for the work on polydisperse systems. The applied research method is quite similar to the method for monodisperse systems. First, we modify the existing code to incorporate the variation of materials' radius. In this case, normal displacement (3) and the list of nearest neighbors (Figure 2) are adjusted dynamically in line with the process of revealing materials' trajectories. Fourth-order Gear Predictor-Corrector method as derived in [2] is also applied to calculate the forces, velocities, and positions of all materials. Second, extensive simulations of deposition process by dropping materials

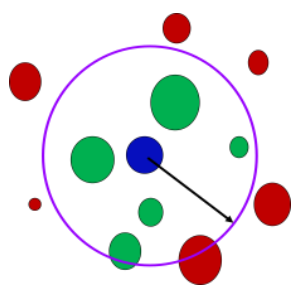

(a)

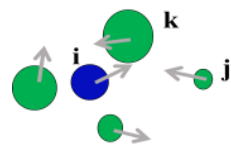

(b)
Figure 2 (a) Cut off distance of nearest neighbor of particle $\mathrm{i}$, and (b) particles ( $\mathrm{j}$ and $\mathrm{k}$ ) in the neighbor list of particle $\mathrm{i}$ that potentially collide with particle $\mathrm{i}$.

one by one from a certain position above the surface and by varying the characteristics of the materials are conducted. Third, some movies are created to investigate the dynamics of the system. Next, we discuss the resulting pile structures and force networks.

\section{RESULTS AND DISCUSSIONS}

The simulations of the deposition process are conducted by consecutively dropping the materials from a fixed vertical position onto the free surface. The surfaces on which the particles are dropped, unless stated otherwise, have the same properties as the materials (discs). Apart from collision forces (1) and (2), the forces acting on a disc also involve gravity, and the total force of disc $\mathrm{i}$ is given by

$\mathbf{f}_{\mathrm{i}}=m \mathbf{g}+\sum_{\text {collisions }}\left(\mathbf{f}_{\mathbf{n i}}+\mathbf{f}_{\mathbf{t}_{\mathrm{i}}}\right)$

with $m$ is the discs mass, $\mathbf{g}$ is the gravitational acceleration.

Throughout the simulations, we use 600 discs of mass $m=5 \times 10^{-2} \mathrm{~kg}$, diameter $\sigma=1.5 \times 10^{-2}-5 \times 10^{-2} \mathrm{~m}$, spring constants in the normal and tangential directions $k_{\mathrm{n}}=k_{\mathrm{t}}=10^{5} \mathrm{kgs}^{-2}$, vertical position $h_{0}=3 \mathrm{~m}$, stick velocity $\varepsilon=10^{-2} \mathrm{~ms}^{-1}$, and time step $\Delta t=10^{-5} \mathrm{~s}$. Other parameter values such as coefficients of friction and restitution are varied. The results are discussed in the following. 


\subsection{Dynamic Behaviuor}

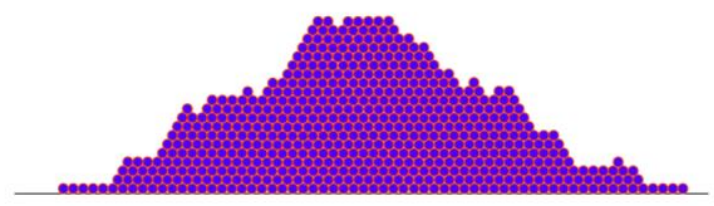

(a)

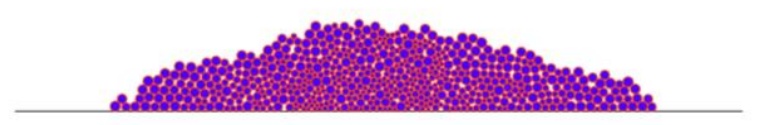

(b)

Figure 3 Simulation results of depositing 600 discs: (a) monodisperse system, and (b) polydisperse system.

In order to observe the behavior of pile formation, some movies are produced using numerical simulation data consisting of a series of consecutive snapshots of the pile configuration. The observations are rather qualitative, but they could be made more quantitative, as in principle the detailed numerical data for each disc is available. It is observed that similar to monodisperse systems, during the pile formation of polydisperse systems, defect lines also develop due to internal stresses, leading to the so-called internal landslides. However, the development of defect lines of the later system does not always form a straight line extending from the surface to the bottom of the pile as observed in the former system. Such mechanism of internal landslides can take place at relatively small fractions of the pile. We also observed that during the deposition process some grains slide down the pile surface. Such phenomenon is called a surface avalanche. Another dynamic mechanism is the tendency of smaller particles to descent in the pile along the holes created by bigger particles. We call this phenomenon an internal arrangement.

For monodisperse systems, repetitive landslides at random points may result in a pile with an irregular surface, resembling a devil's staircase. When the pile grows, the role of surface avalanches increases, which diminishes these irregularities, giving a relatively smooth pile surface. For polydisperse system, the role of internal arrangement leads to flattening the pile surface and hence to a smaller angle of repose. Examples of stable piles on the free surface as found in the simulations are given in Figures 3.

\subsection{Pile Structures}

One can see from Figure 3 that the deposition of discs on free surfaces for both systems produces piles with almost triangular shapes having different surface structures; the surface of polydisperse pile is smoother than that of a monodisperse pile. The steepness of a polydisperse pile, indicated by the angle of repose, depends on the surface roughness of the discs as well as on the medium represented by the coefficient of friction. Increasing surface roughness produces larger angles of repose as shown in Figure 4. From Figure 3, it can also be seen that the local structures of the piles of the two systems are different. The monodisperse pile forms a close-packed structure, while the polydisperse system forms lot of space (voids) along the pile. Such formation is found in the soil texture as studied by Arya-Paris [5] in developing the model of soil pore distribution.

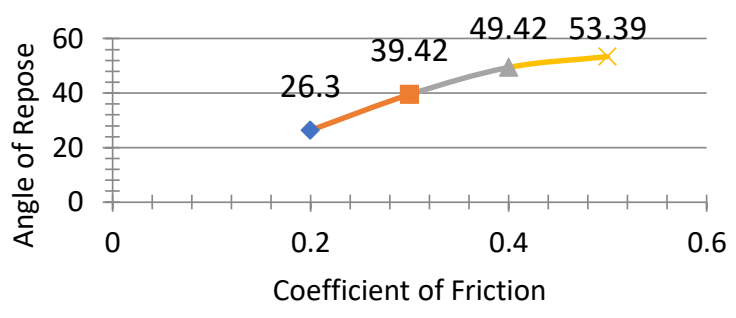

Figure 4 Angle of repose for various coefficient of frictions.

\subsection{Force Networks}

A force network is obtained by drawing line segments between centers of two particles in contact or between the particle and the medium. The line thickness is proportional to the magnitude of compression (normal). The force networks of the piles shown in Figures $3 \mathrm{a}$ and $3 \mathrm{~b}$ are shown in Figures $5 \mathrm{a}$ and $5 \mathrm{~b}$ and represent force networks of monodisperse and polydisperse systems, respectively. It can be seen from Figure 5 that the forces in the pile are not distributed evenly; there are thick and thin lines corresponding to large and small forces. Such phenomenon is due to the fact that the lower layers of stable piles must carry the total weight of the upper part of the pile, hence the average stress per contact increases downwards as expected.

From Figure 5a one sees that the lines of the force network of the monodisperse pile formed are mostly pointing 60 degrees downwards. In many cases the two lines are equally thick, implying the force from the above layers is propagated almost equally to the two underlying discs. In many other cases, the lines are of unequal thickness, and force chains are developing. It can also be observed that there are hardly any horizontal forces, and the force network is composed of diamond-shaped cells. The polydisperse pile, as seen in Figure 5b, shows many bent lines so that almost no force network is pointing at a 
singular angle, leading to a disorder. The many pores within the pile may contribute to such results.

\section{CONCLUSION}

The dynamic mechanisms of a polydisperse pile formation on a free surface are internal landslides, surface avalanches, and internal rearrangement. The resulting pile is almost triangular in shape with quite flat a top. The slope of the triangle (angle of repose) depends on the characteristics of the materials, including the surface roughness of the particles and the medium onto which the particles are deposited. The pile does not form a close-packed structure, leading to the formation of a force network with disordered directions.

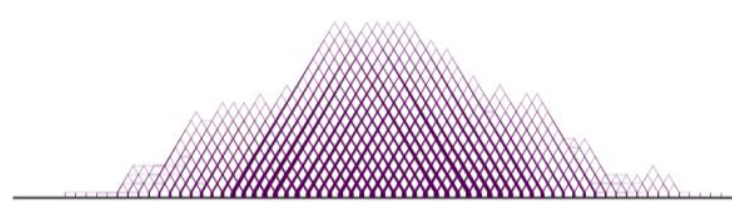

(a)

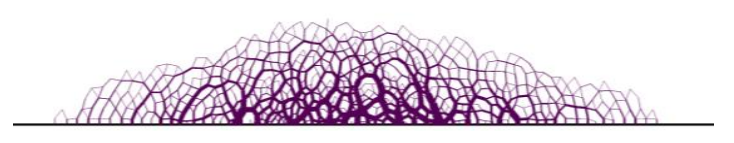

(b)

Figure 5 Force networks of the piles formed on the free surface: (a) monodisperse system, and (b) polydisperse system.

\section{ACKNOWLEDGMENTS}

The author thanks the University of Jember for sponsoring this research.

\section{REFERENCES}

[1] M. Hasan and J.H.J. van Opheusden, "A model for static and dynamic phenomena in deposition processes", J. Indo. Math. Society, vol. 13 (2), pp. 173-189, 2007.

[2] L. Brendel and S. Dippel, "Lasting contacts in molecular dynamics simulations", in Physics of dry granular materials, H.J. Herrmann, J.P. Hovi and S. Luding, Kluwer, Dordrecht, 1998, pp. 313-318.

[3] H.G. Matuttis, S. Luding, and H.J. Herrmann, "Discrete element simulations of dense packing and heaps made of spherical and non-spherical particles", Powder Tech. vol. 109, pp. 278-292, 2000.

[4] M. Hasan and J.H.J. van Opheusden, "Approximations of Coulomb's Law of Friction", Proc. $6^{\text {th }}$ ISSM Global Networking and Research Contributions towards Indonesian Prosperity, pp: 41-45, 2001. ISBN: 0855-8692.

[5] L.M. Arya and J.F. Paris, "A physicoempirical model to predict the soil moisture characteristic from particle-size distribution and bulk density data", Soil Sci. Soc. Am. J. vol. 45, pp.1023-1030, 1981. 